\title{
30-fs 1.6 mJ Pulses at a kHz Repetition Rate from a Single Stage DPSS Yb Amplifier
}

\author{
Giedrius Andriukaitis ${ }^{1}$, Daniil Kartashov ${ }^{1}$, Audrius Pugžlys ${ }^{1}$, Dušan Lorenc ${ }^{1}$, Andrius Baltuška ${ }^{1}$, Linas \\ Giniūnas $^{2}$, Romualdas Danielius ${ }^{2}$, Ömer F. Ilday ${ }^{3}$ \\ ${ }^{1}$ Photonics Institute, Vienna University of Technology, Gusshausstrasse 27-387, A-1040, Vienna, Austria \\ ${ }^{2}$ Light Conversion Ltd., P/O Box 1485, Sauletekio Avenue 10, LT-10223, Lithuania \\ ${ }^{3}$ Department of Physics, Bilkent University, Cankaya, Ankara 06800, Turkey
}

\begin{abstract}
J pulses from a cw-diode-pumped $\mathrm{Yb}: \mathrm{CaF}_{2}$ MOPA are spectrally broadened in Ar and recompressed to $30 \mathrm{fs}$ at $980 \mathrm{~nm}$ using a prism pair. Multi-millijoule 12-fs pulses are feasible upon higher-order spectral phase correction.

(C)2010 Optical Society of America

OCIS codes: $320.5520,140.3615$
\end{abstract}

For over 15 years Ti:sapphire pulse amplifiers emitting $25-150$-fs pulses at $\mathrm{kHz}$ repetition rates have dominated the field of ultrafast applications and ultrashort-pulse laser technology, ultimately leading to the generation of nearsingle-cycle pulses around the wavelength of $800 \mathrm{~nm}$ via external compression. Self-phase modulation (SPM) induced spectral broadening in a gas-filled hollow fiber and laser beam filamentation in gases are well developed techniques for routine production of few-optical-cycle pulses at a sub-mJ energy [1-5]. In this contribution we show encouraging results for millijoule-level pulse broadening and recompression obtained with a novel broadband diodepumped single-stage regenerative 1030-nm Yb:CaF 2 amplifier [6], which holds potential for a robust, compact and cheap (no additional pump lasers for the seeder and the amplifier) alternative to $\mathrm{kHz}$ Ti:sapphire amplifiers.

The layout of the system is presented in Fig. 1. In comparison with the performance of our cw-pumped $\mathrm{Yb}$ laser reported earlier [6], the output energy before the grating compressor was improved to reach $5.5 \mathrm{~mJ}$ at $1 \mathrm{kHz}$ by using a Brewster-cut $\mathrm{Yb}$ crystal. To reduce the system complexity, the deformable mirror in the stretcher was not used this time at the expense of worsening the compressed pulse duration form 170 fs to about $200 \mathrm{fs}$. Because of the high-loss diffraction gratings in the compressor, the energy of the compressed pulse is currently limited to $2.5 \mathrm{~mJ}$ at $1030 \mathrm{~nm}$.

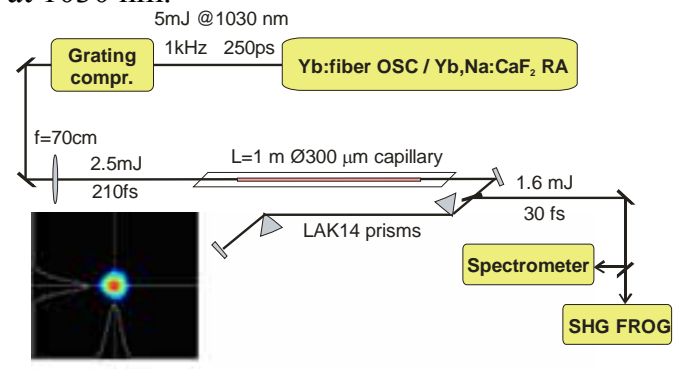

Fig.1: Layout of experimental setup and far-field profile of the hollow fiber output at the Ar pressure of 2.2 bar.

The output beam of a diffraction-limited quality was coupled into a 300- $\mu \mathrm{m}$ diameter, 1-m-long fused silica hollow-core fiber mounted on a V-groove aluminum holder inside of a vacuum tube with 1-mm-thick Brewster windows. Prior to filling it with gas, the tube is evacuated below $10^{-2}$ mbar. In addition to working with the hollowfiber SPM, we also characterized the pulses broadened and mildly self-compressed in a static gas cell when the beam bypasses the capillary inside the gas tube. The spectra recorded at different gas pressures and the corresponding phases, retrieved from SHG FROG measurements are presented in Fig.2a and $2 \mathrm{~b}$ for the cases of a filament in a static gas cell and the $300-\mu \mathrm{m}$ capillary, respectively. The inset to Fig.2a shows an up to $\times 2$ selfcompression of the output pulse occurring at higher Ar pressures, which represents an attractive and easily implementable low-loss pulse shortening add-on for the regenerative amplifier.

Owing in part to the outstanding input beam quality, the throughput of the hollow fiber was $65 \%(\sim 1.6 \mathrm{~mJ})$ and remained nearly constant with gas pressure. Starting with the Ar pressure of 2.4 bar, white light generation from the walls of the glass capillary was observed, which prevented us from operating at higher pressures. Spectra supporting a 12-fs transform-limited pulse duration were recorded at 2.2 bar. The corresponding chirped pulse carries a reasonably-behaved phase, as revealed by the corresponding FROG measurements (Fig. 2b-d). The pulses were partially recompressed to $30 \mathrm{fs}$ FWHM using a low-loss prism compressor (a pair of $60^{\circ}$ LAK14 prisms). The corresponding results of SHG FROG characterization are given in Fig. 3. Note that in order to avoid phase excursions in the spectral wings, where the prism compressor runs into higher-order phase correction problems, the pulse spectrum was deliberately narrowed by lowering the Ar pressure to 2.0 bar. Therefore, despite a significantly 


\section{CThA2.pdf}

narrower gain bandwidth than that of Ti:sapphire, the $\mathrm{Yb}$ laser is capable of delivering similar energy and pulse duration specifications as broadband Ti:sapphire amplifiers.
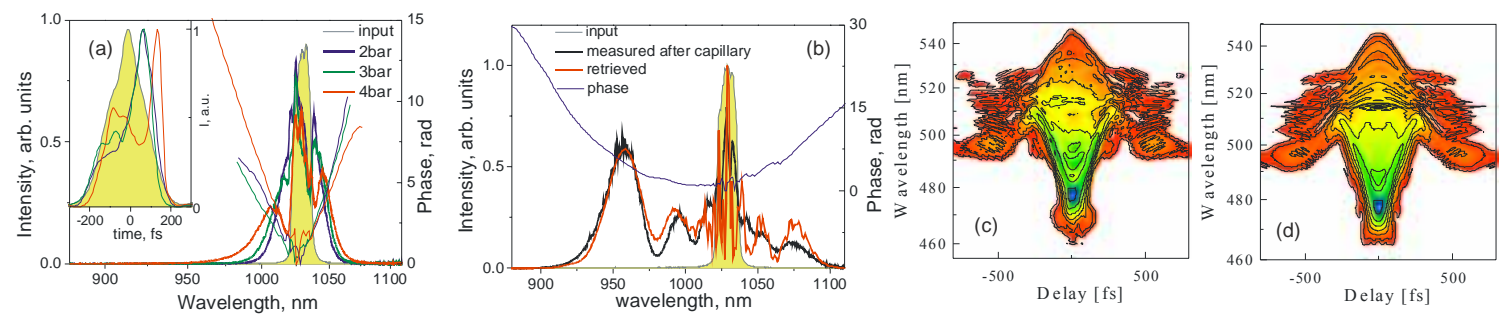

Fig. 2: SHG FROG characterization of chirped pulses. Spectral intensity and phase after a filament in an (a) Ar-filled cell and (b) hollow fiber at 2.2 bar of Ar. (c) measured and (d) reconstructed SHG FROG traces after a 2.2-bar hollow fiber.

The developed system has large potential for scaling up the energy of the compressed pulses. This potential originates from a substantially longer wavelength, in comparison with widely used Ti:Sapphire-based femtosecond laser systems. The intensity of a laser pulse which can be successfully transported and spectrally broadened in a gasfilled hollow fiber is limited by ionization of the gas. In spite of attempts to use the ionization nonlinearity for spectral broadening of pulses at a multi-mJ energy level [7,8], SPM in gases is still the most controllable and reliable technique for ultrashort pulse production. The inner diameter of a hollow-core waveguide is limited by coupling and maintaining of the fundamental mode of the waveguide when a laser pulse propagates through. Thus, the maximum energy in a laser pulse which can be realistically transmitted and spectrally broadened for the Ti:Sapphire wavelength of $800 \mathrm{~nm}$ is $\leq 1 \mathrm{~mJ}$ for a $250-300 \mu \mathrm{m}$ capillary filed by Ne. In our system we can use capillaries with a diameter up to 400-450 $\mu \mathrm{m}$ which, with using Ne, will allow an increase in energy of compressed pulses of a factor of 4-5 at least.
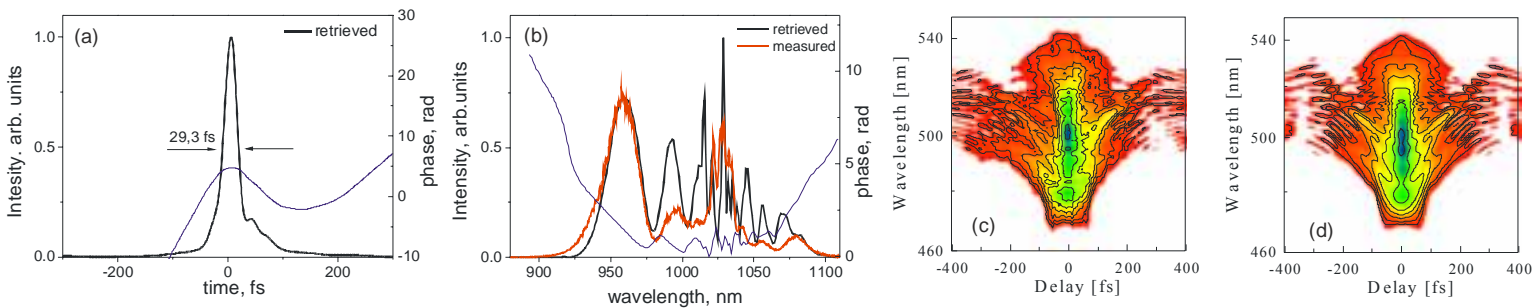

Fig. 3: Partial recompression to $30 \mathrm{fs}$ with a LAK14 prism pair of the pulse transmitted through the hollow fiber at 2.0 bar of Ar. (a) reconstructed temporal intensity and phase. (b) spectral intensity and phase. (c) measured and (d) reconstructed SHG FROG traces.

In conclusion, we demonstrated for the first time efficient pulse compression at millijoule energy level of the output of the $200 \mathrm{fs}, 1 \mathrm{kHz}$, cw-diode-pumped $\mathrm{Yb}: \mathrm{CaF}_{2} \mathrm{MOPA}$, using spectral broadening in a gas-filled hollow-core fiber and the simplest prism compressor. This system has large potential for further energy scaling of the compressed pulses and can compete with similar energy level Ti:sapphire laser amplifiers over which, owing to direct diode pumping, it holds the edge in terms of robustness, dependability, and simplicity.

\section{References}

[1] M. Nisoli, S. De Silvestri, and O. Svelto "Generation of high energy 10 fs pulses by a new pulse compression technique", Appl. Phys. Lett. 68, 2793-2795 (1996).

[2] M. Nisoli et al. "Compression of high-energy laser pulses below 5 fs", Opt. Lett. 22, 522-524 (1997).

[3] G. Stibenz, N. Zhavoronkov, and G. Steinmeyer "Self-compression of millijoule pulses to 7.8 fs duration in a white-light filament", Opt. Lett. 31, 274-276 (2006).

[4] C.P. Hauri et al. "Intense self-compressed, self-phase-stabilized few-cycle pulses at $2 \mu \mathrm{m}$ from an optical filament", Opt. Lett. 32, 868-870 (2007).

[5] A. Zaïr et al. "Spatio-temporal characterization of few-cycle pulses obtained by filamentation", Opt. Exp. 15, 5394-5405 (2007).

[6] A. Pugžlys et al. "Multi-mJ, 200 fs, cw-pumped, cryogenically cooled, Yb, Na:CaF 2 amplifier", Opt. Lett. 34, $2075-2077$ (2009).

[7] A. Suda et al. "Generation of sub-10-fs, 5-mJ-optical pulses using a hollow fiber with a pressure gradient", Appl. Phys. Lett. 86, 111116(1-3) (2005).

[8] A. Babin et al. "Compression of High-Intensity Femtosecond Laser Pulses Due to Ionization Self-Phase Modulation in Gas-Filled Capillary Tubes”, Las. Phys. 13, 1050-1053 (2003). 